\title{
Effective thermal conductance of thermoelectric generator modules
}

\author{
I. Ruiz ${ }^{1}$, M. Borrelli ${ }^{2}$, T. Pujol ${ }^{1}$, N. Luo ${ }^{1}$, L. Pacheco ${ }^{1}$, A. Massaguer ${ }^{1}$, L. Montoro ${ }^{1}$ \\ ${ }^{1}$ Polytechnic School, University of Girona, Campus Montilivi, 17071 Girona (Spain) \\ ${ }^{2}$ Università degli Studi del Sannio, Piazza Roma, 21, 82100 Benevento (Italy) \\ Corresponding author: T. Pujol, phone number: +0034 972 418865, e-mail: toni.pujol@udg.edu
}

\begin{abstract}
Recent studies have analyzed the viability of generating electricity by means of thermoelectric modules applied to processes with large amounts of waste heat. The simulation of the performance of large-scale designs of thermoelectric generators (TEGs), however, is a very complex task since it involves a coupling between both electrical and thermal phenomena. In addition, a single TEG module contains tenths of small P-type and $N$-type semiconductor legs, which implies that the simulation must take into account domains with characteristic lengths that vary several orders of magnitude. Here, we propose a methodology for determining the effective heat conductance of a single TEG module that can be employed for simulating the entire element without entry into the details of its inner composition. The effective thermal conductance here proposed can be understood as the coefficient that predicts, at different operating conditions, an upper bound of the electrical power generated by the TEG module.
\end{abstract}

\section{Key words}

Thermoelectric generator, TEG module, Waste heat recovery.

\section{Introduction}

The use of thermoelectric generators (TEGs) for recovering waste heat and converting it into electricity has been extensively analyzed during the last years [1]. The advances on thermoelectric materials, with higher figures of merit at lower temperatures, and the reduction of the cost per TEG module, have increased the commercial interest in this technology [2]. Several large-scale designs have been proposed, mainly focused on extracting energy from exhaust gases of internal combustion engines [3].

However, the simulation of the performance of large-scale TEG systems requires a high computational effort since both thermal and electrical processes are coupled and the characteristic scale at the $P$-type and $N$-type semiconductor level is several orders of magnitude smaller than that corresponding to the whole design. Simulations of the thermoelectric behavior are usually applied to a single pair of $P$-type and $N$-type material [4] (see Fig. 1), with very few attempts to simulate a single TEG module since it may contain $n$ (> 100) pairs of $P$-type and $N$-type legs (see Fig. 1) [5].

Therefore, the task of simulating the behavior of a full TEG device that contains tenths of TEG modules is often carried out by assuming the same properties for the $n$ pairs of $P$ and $N$ semiconductor legs (electrically connected in serial and thermally connected in parallel configuration) in each one of the modules analyzed [6]. This assumption leads to the definition of global parameters for the TEG module [7] that may be used for modeling its behavior. However, some manufacturers of TEG modules do not provide enough information for deducing the values of the global parameters at different temperatures, with public data related to the point of maximum power at the goal temperature gradient between the hot and cold junction. The extrapolation of the global properties obtained at the maximum power to scenarios with smaller temperature gradients may substantially overestimate the net electrical production of the module.
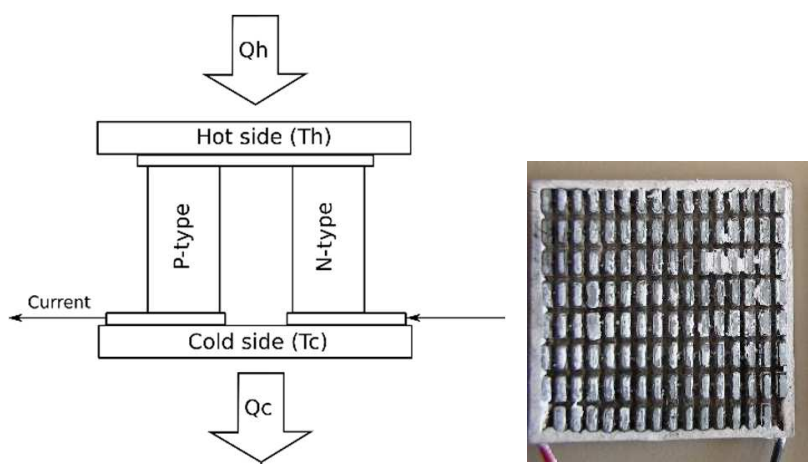

Fig. 1. Scheme of a pair of $P$-type and $N$-type elements of a TEG (left). Distribution of $n$ pairs of $P$-type and $N$-type elements in the TEG analyzed in the present paper (right).

Therefore, here we propose a methodology in order to calculate the main parameters of the TEG module as a function of temperature. This will allow us to define a global effective thermal conductance for the whole module specifically fitted to satisfy the condition of maximum electrical power output at any temperature gradient. 


\section{TEG model}

When a TEG module is connected to an external load $R_{L}$, see Fig. 2, the heat flux applied generates an electrical current.

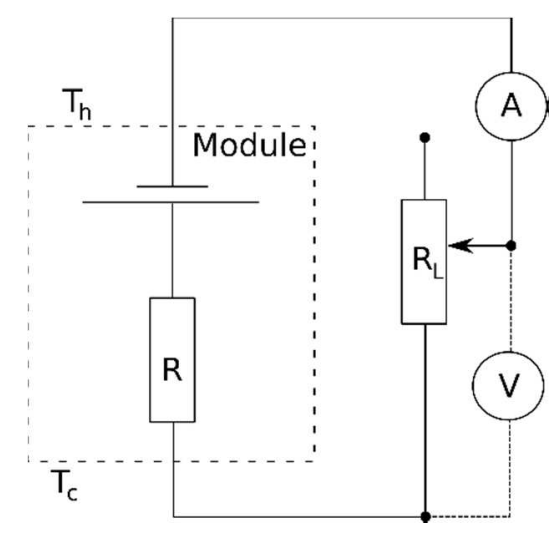

Fig. 2. Electrical scheme of a TEG module connected to a variable load $\left(R_{L}\right)$. A measures the electrical current $I$ and $V$ the voltage $V$ at both ends of the TEG module.

Both thermal and electrical effects are related by means of the equations that model the rate of heat flow to the hot junction $Q_{h}$ and from the cold junction $Q_{c}$ :

$$
\begin{aligned}
& Q_{h}=\alpha T_{h} I-\frac{R I^{2}}{2}+K\left(T_{h}-T_{c}\right) \\
& Q_{c}=\alpha T_{c} I+\frac{R I^{2}}{2}+K\left(T_{h}-T_{c}\right)
\end{aligned}
$$

where $T_{h}$ is the hot junction temperature, $T_{c}$ is the cold junction temperature, $I$ is the current, $R$ is the inner electrical resistance, $K$ is the inner thermal conductance and $\alpha$ is the Seebeck coefficient.

The electrical power output $P$ of the TEG module follows:

$$
P=Q_{h}-Q_{c}=\alpha\left(T_{h}-T_{c}\right) I-R I^{2}
$$

with a thermal efficiency $\eta$ equal to:

$$
\eta=\frac{P}{Q_{h}}
$$

For open-circuit conditions $\left(R_{L}=\infty\right)$, the current intensity $I$ $=0 \mathrm{~A}$ and the voltage reaches its maximum value. For shortcircuit conditions $\left(R_{L}=0 \Omega\right)$, the current intensity $I$ is maximum and the voltage is minimum $(=0 \mathrm{~V})$. In both situations the power output $P$ is null. The power output is maximum $\left(P_{\max }\right)$ when the external load matches the inner value (i.e, with $R_{L}=R$ ), with $P_{\max }$ equal to:

$$
P_{\max }=\frac{\alpha^{2}\left(T_{h}-T_{c}\right)^{2}}{4 R}
$$

being achieved when the current is:

$$
I_{P, \max }=\frac{\alpha\left(T_{h}-T_{c}\right)}{2 R}
$$

that is half the maximum intensity value (that at $R_{L}=0 \Omega$ ). From Equations (1)-(2), the mean heat transfer through the TEG module is:

$$
Q_{\text {mean }}=\frac{Q_{h}+Q_{c}}{2}=\alpha T_{\text {mean }} I+K\left(T_{h}-T_{c}\right)
$$

where:

$$
T_{\text {mean }}=\frac{T_{h}+T_{c}}{2}
$$

is the mean temperature between the hot and cold faces of the module.

The effective thermal conductance $K_{\text {eff }}$ for the TEG module is defined as:

$$
Q_{\text {mean }}=K_{e f f}\left(T_{h}-T_{c}\right)
$$

Here, we use the $K_{\text {eff }}$ value for the external load that produces the maximum power. Thus, substituting Eq. (6) into Eq. (7) and with Eq. (9), we obtain:

$$
K_{\text {eff }}=\frac{\alpha^{2} T_{\text {mean }}}{2 R}+K
$$

since the dimensionless figure of merit $Z \bar{T}$ is defined, as usual, as:

$$
Z \bar{T}=\frac{\alpha^{2}}{R K} T_{m e a n}
$$

the effective thermal conductance $K_{\text {eff }}$ reads:

$$
K_{e f f}=K\left(1+\frac{Z \bar{T}}{2}\right)
$$

Equation (12) provides a value of the thermal conductance of the entire module that may be used for estimating an upper bound of the maximum power output of TEG devices under steady state conditions. Tabulated values of $K$ and $Z \bar{T}$ as a function of the mean temperature across the module can be employed in order to set the temperature dependence of Eq. (12). Once done, the use of Eq. (9) for expressing the thermal behavior of the whole TEG module (instead of Eqs. (1) and (2)) will provide a fast solution of the thermal equations since, now, they are not coupled with the electrical terms. Once solved, the estimated power per module can be calculated with Eq. (5) (that also ignores the effect of serial-parallel connections between modules). By doing so, however, we accept that the external load matches, at any temperature, the inner module resistance $R$, implying that the calculation of the power output will correspond to the maximum value that the device can achieve.

The use of $K_{\text {eff }}$, however, may lead to large discrepancies between the power output predicted and the actual one when using values of $K$ and $Z \bar{T}$ independent of 
temperature and equal to those of the optimum conditions published by the manufacturer. In order to obtain a reasonable upper bound of the power output by employing the $K_{\text {eff }}$ value, it is needed to correctly determine the temperature dependence of the global parameters of the TEG module: thermal conductance $K$, Seebeck coefficient $\alpha$ and inner resistance $R$. In the next section, we detail the experimental set up carried out for measuring these parameters.

\section{Experimental set up}

We have analyzed the TEG126-40B module of Everredtronics. The features of this module published by the manufacturer are listed in Table I.

Table I. - Specifications of the TEG126-40B module for $T_{h}=$ $300^{\circ} \mathrm{C}$ and $T_{c}=50^{\circ} \mathrm{C}$

\begin{tabular}{|l|c|c|}
\hline \multicolumn{1}{|c|}{ Description } & Symbol & Value \\
\hline Length & $L$ & $40 \mathrm{~mm}$ \\
\hline Width & $W$ & $40 \mathrm{~mm}$ \\
\hline Seebeck coefficient & $\alpha$ & $0.02667 \mathrm{~V} \mathrm{~K}^{-1}$ \\
\hline Open-circuit voltage & $V_{o c}$ & $6.67 \mathrm{~V}$ \\
\hline Matched load resistance & $R$ & $1.7 \Omega$ \\
\hline Matched output voltage & $V_{P \max }$ & $3.58 \mathrm{~V}$ \\
\hline Matched output current & $I_{\max }$ & $1.9 \mathrm{~A}$ \\
\hline Matched output power & $P_{\max }$ & $6.8 \mathrm{~W}$ \\
\hline $\begin{array}{l}\text { Rate of heat flow through } \\
\text { module }\end{array}$ & $Q_{h}$ & $137.0 \mathrm{~W}$ \\
\hline
\end{tabular}

From Table I, under conditions of $T_{h}=300^{\circ} \mathrm{C}$ and $T_{c}=50^{\circ} \mathrm{C}$ at matched load resistance (i.e., with an external load that maximizes the power), the net efficiency of the TEG module reaches $\eta_{m}=4.9 \%$, the thermal conductance $K_{m}=$ $0.444 \mathrm{~W} \mathrm{~K}^{-1}$ and the figure of merit is $Z \bar{T}_{m}=0.42$. However, at a temperature gradient smaller than $T_{h}-T_{c}=250^{\circ} \mathrm{C}$, the values of the above parameters may substantially differ. Therefore, the global parameters of the module obtained at different temperatures have been experimentally obtained with the set up shown in Fig. 3.

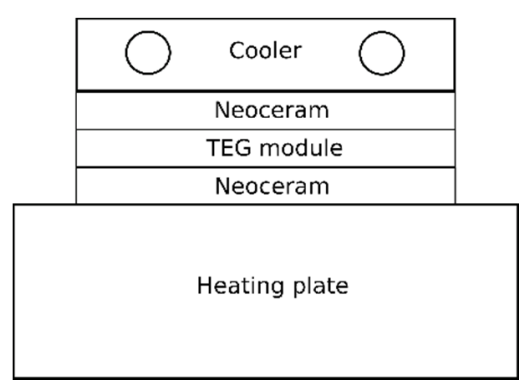

Fig. 3. Schematic of the experimental set up. In experiments with high $\Delta T$, the neoceram slab located at the top is removed and the TEG module makes contact with the water cooler. Temperature is recorded in all contact faces.

The experimental set up consists of a heating resistance electronically controlled that heats a pack formed by the TEG module located between two slabs of neoceram, a transparent ceramic glass with known heat conductivity. Each piece of neoceram is $4 \mathrm{~mm}$ thick, $40 \mathrm{~mm}$ width and $40 \mathrm{~mm}$ length. The cold side uses an aluminum water cooling element of dimensions $40 \mathrm{~mm} \times 40 \mathrm{~mm}$ with water entry at $18^{\circ} \mathrm{C}$. Water outlet temperature has not been recorded. Sheets made of brass with dimensions $40 \mathrm{~mm} \mathrm{x}$ $40 \mathrm{~mm}$ and $0.2 \mathrm{~mm}$ thick are located between all contact elements. These sheets have a small cut that reaches the center of the geometry in which we have introduced Ktype thermocouples of diameter $0.2 \mathrm{~mm}$ for measuring the temperature at the faces. The change of thermal conductivity of brass with temperature has little effect in our results since we focus only on recording cases with constant temperature difference across the TEG for a fixed value of the hot temperature. Temperature data are acquired with a National Instruments module and the Labview software.

In scenarios with a high demand of cooling, the neoceram in between the cooler and the TEG module is removed, so the cold junction of the TEG module is directly in contact with the aluminum cooler.

The electric circuit consists of an external, purely resistive and variable load $R_{L}$ with a maximum value of $5.2 \Omega$. Voltage and intensity have been measured following the scheme shown in Fig. 2 by means of National Instruments modules and the LabView software.

The goal of the experiment consists of recording the measured variables for a wide range of the external load values $R_{L}$ keeping constant the temperature difference $T_{h}$ $-T_{c}$ across the TEG module for a fixed value of the hot temperature $T_{h}$.

Thus, we first set a target value of $T_{h}$ and, with no load, obtained the $T_{h}-T_{c}$ across the TEG module. Once the system has reached steady state conditions for a minimum time period of 4 minutes, the value of the external load resistance is increased (at $0.5 \Omega$ intervals) and the experiment is repeated. Slight changes in the water mass flow (cooler) and the electronically controlled heater may be required for maintaining the values of $T_{h}$ and $T_{h}-T_{c}$. The entire process is repeated 6 times for a different target value of $T_{h}$. The measured data are temperature values $T$ at all interfaces, intensity $I$, voltage $V$ and external load resistance $R_{L}$. Relative errors are below $2 \%$ for all data.

\section{Results}

\section{A. Power and efficiency}

The electrical power is calculated from the measured data $I$ and $V$ :

$$
P=V I
$$

and the values obtained, as a function of the external load $R_{L}$ are shown in Fig. 4 for different values of $\Delta T=T_{h}-T_{c}$. In Fig. 4, sd means the standard deviation of the $\Delta T$ measured data for the array of values recorded during a time interval of 4 minutes. Note from Fig. 4 that the maximum power shifts to higher values of the external load as $\Delta T$ increases. 


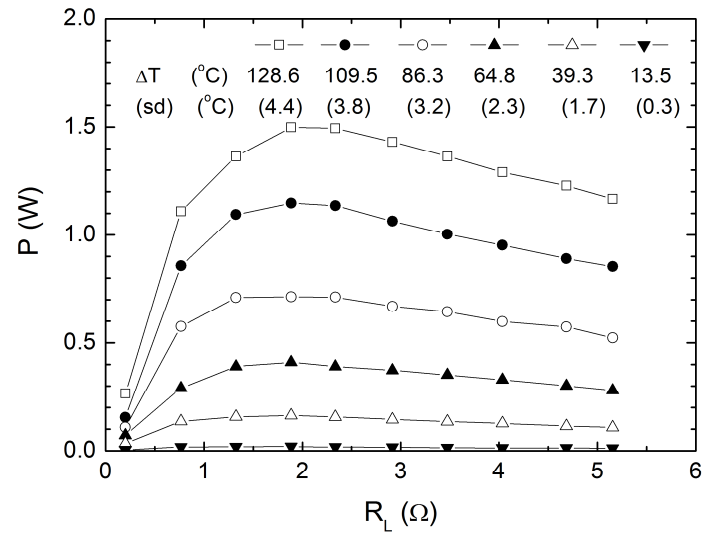

Fig. 4. Power $P$ as a function of the external load $R_{L}$ for different values of $\Delta T$. sd means the standard deviation of the $\Delta T$ series of data. Manufacturer's data, $P=6.8 \mathrm{~W}$ at $R=1.7 \Omega, \Delta T=250^{\circ} \mathrm{C}$.

This shift is also observed in Fig. 5, where the net efficiency as a function of the external load is shown. As expected, the external load that produces the maximum efficiency does not coincide with the value that provides the maximum power [8]. Note that all values obtained for the net efficiency are below $2 \%$, being much smaller than the corresponding figure provided by the manufacturer under the expected working conditions: $\eta=4.9 \%$ at $\Delta T=250^{\circ} \mathrm{C}$.

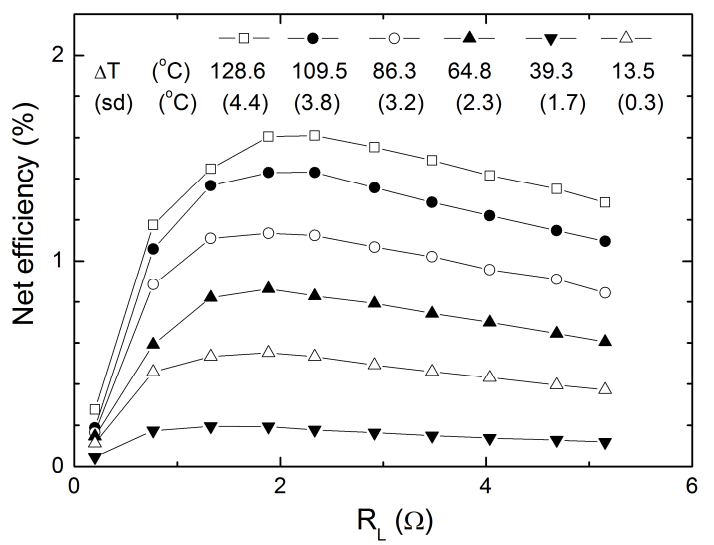

Fig. 5. Net efficiency $\eta$ as a function of the external load $R_{L}$ for different values of $\Delta T$. sd means the standard deviation of the $\Delta T$ series of data. Manufacturer's data, $\eta=4.9 \%$ at $\Delta T=250{ }^{\circ} \mathrm{C}$.

We note that for calculating the net efficiency, the value of the rate of heat flow $Q_{h}$ must be known in Eq. (4). This has been assumed to be equal to the rate of heat flow calculated from the known thermal conductivity of the neoceram at the hot side (see Fig. 3) and the recorded temperatures at its hot and cold sides. This values range from $107 \mathrm{~W}$ for the $\Delta T=$ $128.6^{\circ} \mathrm{C}$ (open squares) to only $9 \mathrm{~W}$ for the $\Delta T=13.5^{\circ} \mathrm{C}$ case (inverted triangles).

\section{B. Seebeck coefficient and inner resistance}

By substituting Eq. (13) into Eq. (3), we obtain

$$
V=\alpha\left(T_{h}-T_{c}\right)-R I
$$

In our experiments, $\Delta T=T_{h}-T_{c}$ is constant, so a linear regression of $V$ vs $I$ predicts the value of the Seebeck coefficient $\alpha$ and the inner resistance $R$. Figure 6 shows the relationship between voltage and intensity measured when changing the external load for different values of $\Delta T$. Linear regressions applied to each series of $\Delta T$ data give correlation coefficients $r>0.998$ for all cases. The coefficients of the fitted linear equation are compared with Eq. (14) and, hence, the inner resistance (minus the slope of the linear fit) and the Seebeck coefficient are obtained. The results are shown in Figs. 7 and 8 where we have plotted the values as a function of $\Delta T$.

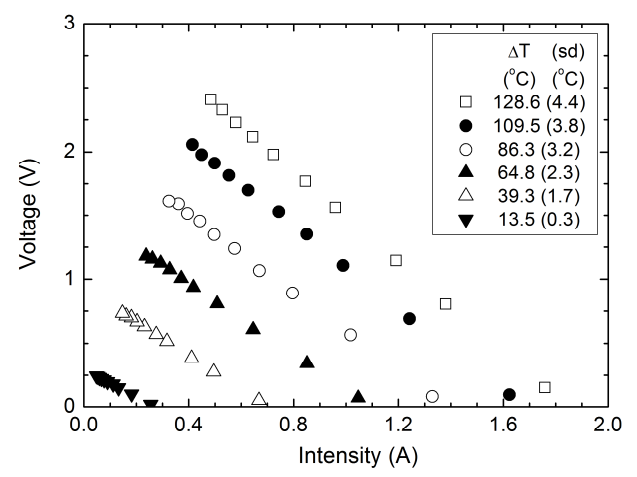

Fig. 6. Voltage as a function of the intensity when changing the external load $R_{L}$ for different values of $\Delta T=T_{h}-T_{c}$. sd means the standard deviation of the $\Delta T$ series of data.

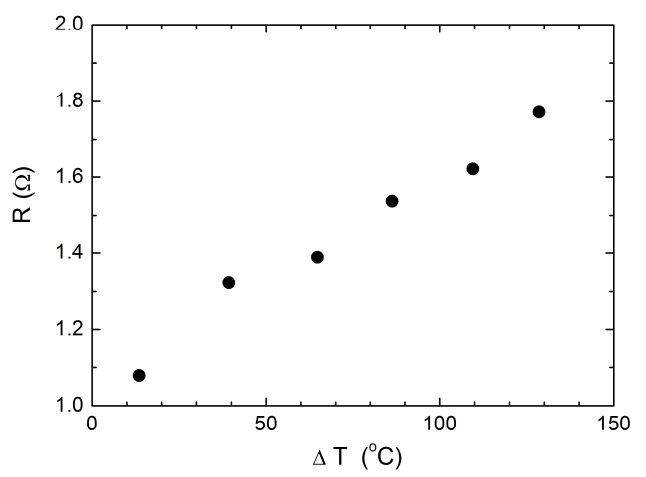

Fig. 7. Inner resistance as a function of $\Delta T$ derived from Fig. 6 . From manufacturer's data, $R=1.7 \Omega$ at $\Delta T=250^{\circ} \mathrm{C}$.

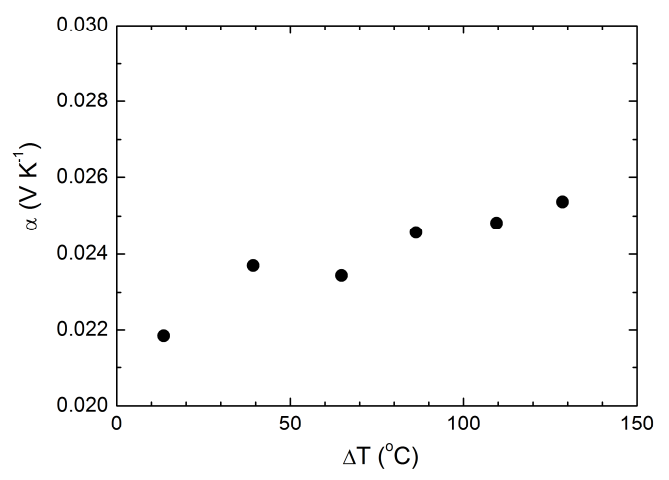

Fig. 8. Seebeck coefficient as a function of $\Delta T$ derived from Fig. 6. From manufacturer's data, $\alpha=0.02667 \mathrm{~V} \mathrm{~K}^{-1}$ at $\Delta T=$ $250^{\circ} \mathrm{C}$. 
Note from Figs. 7 and 8 that these global coefficients substantially depend on temperature, so the use of constant values when simulating the behavior of the whole module is not recommended. In comparison with the manufacturer's data (provided at a single point of functioning, see Table I), the extrapolation of the expected trend reveals a slight discrepancy for the inner resistance value whereas it predicts a very similar value for the Seebeck coefficient. We point out that our experiment has not reached the temperature conditions proposed by the manufacturer due to limitations in the experimental set up.

\section{Maximum power}

The maximum power predicted by Eq. (5) with the experimentally calculated values of the Seebeck coefficient $\alpha$ (Fig. 8) and the inner resistance $R$ (Fig. 7) is shown in Fig. 9 . These values remarkably match the peak values shown in Fig. 4.

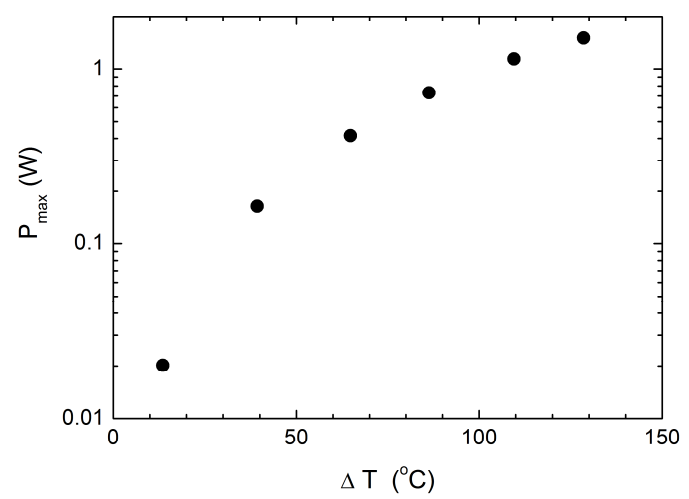

Fig. 9. Maximum power predicted from Eq. (5) with the values of $\alpha$ and $R$ found experimentally. From manufacturer's data, $P_{\max }=6.8 \mathrm{~W}$ at $\Delta T=250{ }^{\circ} \mathrm{C}$.

\section{Thermal conductance}

From Eq. (1), the thermal conductance $K$ of the thermoelectric module can be estimated from:

$$
K=\frac{Q_{h}-\alpha T_{h} I+R I^{2} / 2}{T_{h}-T_{c}}
$$

In our experimental set up, the value of $Q_{h}$ is always known and estimated to be equal to that calculated from the hot neoceram slab (see Fig. 3). Since the other variables used in the right-hand-side of Eq. (15) are also measured or calculated from experimental data (like, for example, $\alpha$ and $R$ ), the thermal conductance can be easily obtained as a function of the external load. The results are shown in Fig. 10 and indicate a trend of decreasing the thermal conductance $K$ as the temperature difference through the module increases. At a given value of the temperature gradient, the results of $K$ slightly varies, with some discrepancies at low values of the external load. The mean value of the thermal conductance for each one of the data series shown in Fig. 10 are depicted in Fig. 11. In this figure, the error bars correspond to one standard deviation for each one of the data series shown in Fig. 10.

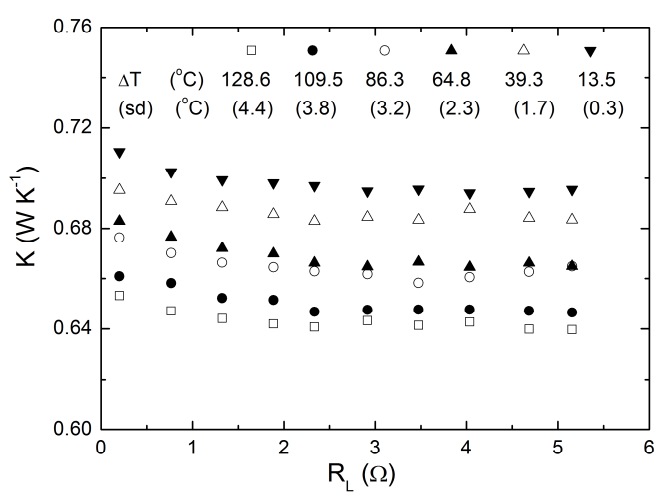

Fig. 10. Thermal conductance $K$ as a function of the external load $R_{L}$.

In the range of temperature gradients analyzed in the present paper, the thermal conductance $K$ substantially decreases as a function of the temperature gradient, as seen in Fig. 11. Therefore, the assumption of using the $K$ value calculated at the operating conditions suggested by the manufacturer (Table I) would overestimate the temperature gradient achieved in scenarios different than the optimum one and, at the same time, the power obtained by the module.

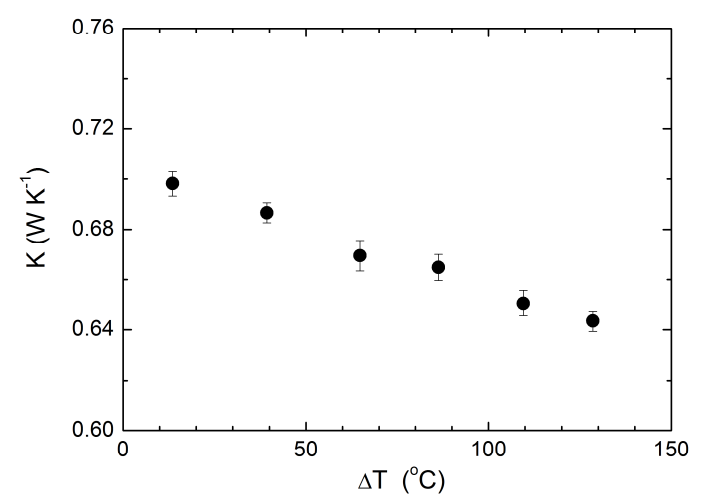

Fig. 11. Mean thermal conductance $K$ as a function of the temperature gradient. From manufacturer's data, $K=0.444 \mathrm{~W}$ $\mathrm{K}^{-1}$ at $\Delta T=250^{\circ} \mathrm{C}$.

\section{E. Effective thermal conductance}

Finally, the effective thermal conductance $K_{\text {eff }}$ for the entire TEG module is calculated after applying Eq. (10) with the results of $K, \alpha$ and $R$ previously obtained. The results are shown in Fig. 12. In comparison, the values of $K_{\text {eff }}$ experimentally obtained after applying Eq. (9) with the measured heat flux value differ less than $2.5 \%$.

Note that the $K_{\text {eff }}$ value shown in Fig. 12 corresponds to the thermal conductance reached by the module when the intensity and voltage correspond to those of the maximum power output (not maximum efficiency) at a given $\Delta T$ value. This effective thermal conductance (as a function of temperature) may be employed for modeling the overall 
thermal behavior of the TEG module once linked to other elements that may form the TEG device. Once the temperature difference $\Delta T$ across the module has been obtained, the power output may be estimated by employing the data shown in Fig. 9. This method overestimates the power obtained since it assumes that the external load is always tuned to maximize the energy generation. However, it is more realistic than extrapolating the characteristic thermoelectric coefficients at the suggested operating conditions of the manufacturer to other ranges of temperature gradients.

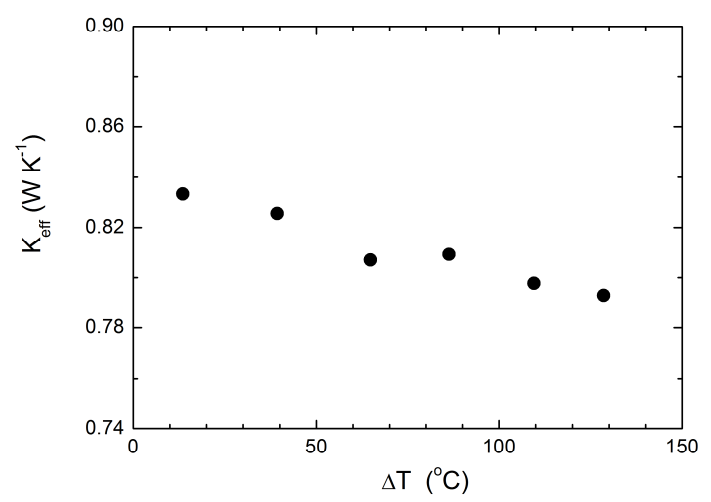

Fig. 12. Effective thermal conductance $K_{\text {eff }}$ as a function of $\Delta T$.

The ratio between the effective thermal conductance $K_{\text {eff }}$ and the thermal conductance $K$ may be employed to estimate the value of $Z \bar{T}$ at different temperature gradients. From Eq. (12), values of $Z \bar{T}$ range from 0.49 to 0.39 , being similar to the 0.42 value provided by the manufacturer.

\section{Conclusions}

It is common that the design of TEG devices involves the simulation of fluid flows that either supply or absorb heat to both cold and hot sides of the system. The latter, at the same time, is composed of multiple elements, including several commercial TEG modules. The modeling of this problem is especially complex when trying to include the coupled effects between the thermal and the electrical phenomena at the thermoelectric level. However, the virtual substitution of TEG modules with slabs of equivalent thermal properties must be done with extreme caution since the results may substantially overestimate the power output.

Here, we have developed a methodology for determining the effective thermal conductance of a commercial thermoelectric module. The main contributions of the methodology employed are: 1) the effective thermal conductance is a function of temperature and 2) its value corresponds to the condition that, for any temperature gradient, provides the maximum electrical power output. The latter point implies that only the thermal analysis is required, which substantially simplifies the numerical solution. Once done, the electrical power output can be easily estimated by taking into account the temperature difference simulated across the TEG module and by applying the conditions of maximum power. Therefore, this methodology provides an upper bound of the maximum power output that may be extracted from a TEG device.
Finally, further improvements may be applied to this methodology. For example, the assumption that $T_{h}$ and $T_{c}$ are the actual temperatures at the hot and cold junctions of both $P$-type and $\mathrm{N}$-type semiconductor legs breaks down when including the conductivity of the ceramic, solders, and metal strips that form the TEG module. In this case, a corrected expression of the Seebeck coefficient, among other modifications, may be employed (see, e.g., [9]). Future work will be devoted to carry out experimental studies with large TEG devices in order to determine the validity of the methodology here proposed.

\section{Acknowledgement}

This work has been partially funded by the University of Girona under grant MPCUdG2016-4.

\section{References}

[1] D. M. Rowe, "Thermoelectrics, an environmentallyfriendly source of electrical power", Renewable Energy 1999, Vol. 16, pp. 1251-1256.

[2] L.I. Anatychuk and R. V. Kuz, "Materials for vehicular thermoelectric generators", Journal of Electronic Materials 2012, Vol. 41, pp. 1778-1784.

[3] M. Hatami, D.D. Ganji and M. Gorji-Bandpy, "A review of different heat exchangers designs for increasing the diesel exhaust waste heat recovery", Renewable and Sustainable Energy Reviews 2014, Vol. 37, pp. 168-181.

[4] W-H. Chen, S-R. Huang and Y-L. Lin, "Performance analysis and optimum operation of a thermoelectric generator by Taguchi method", Applied Energy 2015, Vol. 158, pp. 44-54.

[5] O. Högblom and R. Andersson, "Analysis of thermoelectric generator performance by use of simulations and experiments", Journal of Electronic Materials 2014, Vol. 43, pp. 2247-2254.

[6] Y.Y. Hsiao, W.C. Chang and S.L. Chen. "A mathematic model of thermoelectric module with applications on waste heat recovery from automobile engine", in Energy 2010, Vol. 35, pp. 1447-1454.

[7] M.H. Elsheikh, D.A. Shnawah, M.F. Mohd Sabri, S. B. Mohd Said, M. H. Hassan, M. B. Ali Bashir and M. Mohamad, "A review on thermoelectric renewable energy: Principle parameters that affect their performance", Renewable and Sustainable Energy Reviews 2014, Vol. 30, pp. 337-355.

[8] J. M. Gordon, "Generalized power versus efficiency characteristics of heat engines: Ther thermoelectic generator as an instructive illustration", American Journal of Physics 1991, Vol. 59, pp. 551-555.

[9] C-T. Hsu, G-Y. Huang, H-S. Chu, B. Yu and D-J. Yao, "An effective Seebeck coefficient obtained by experimental results of a thermoelectric generator module", Applied Energy 2011, Vol. 88, pp. 51735179. 